\title{
Author Correction: Best practices for benchmarking germline small-variant calls in human genomes
}

Peter Krusche, Len Trigg, Paul C. Boutros (D), Christopher E. Mason (D), Francisco M. De La Vega (D), Benjamin L. Moore (D), Mar Gonzalez-Porta, Michael A. Eberle, Zivana Tezak, Samir Lababidi, Rebecca Truty, George Asimenos, Birgit Funke, Mark Fleharty, Brad A. Chapman, Marc Salit, Justin M. Zook (D) and the Global Alliance for Genomics and Health Benchmarking Team

Correction to: Nature Biotechnology https://doi.org/10.1038/s41587-019-0054-x, published online 11 March 2019.

In the version of this article initially published online, two pairs of headings were switched with each other in Table 4: "Recall (PCR free)" was switched with "Recall (with PCR)," and "Precision (PCR free)" was switched with "Precision (with PCR)." The error has been corrected in the print, PDF and HTML versions of this article. 